\title{
Numerical evaluation of multi-loop integrals using subtraction terms
}

\author{
A. Freitas \\ Pittsburgh Particle-physics Astro-physics \& Cosmology Center (PITT-PACC), \\ Department of Physics \& Astronomy, University of Pittsburgh, Pittsburgh, PA 15260, USA
}

\begin{abstract}
A formalism for the numerical integration of one- and two-loop integrals is presented. It is based on subtraction terms which remove the soft, collinear and some of the ultraviolet divergences from the integrand. The numerical integral is performed in the Feynman parameter space, using a complex contour deformation to ensure robust convergence even in the presence of physical thresholds. The application of the proposed procedure is demonstrated with several one- and two-loop examples. An implementation in the program NICODEMOS is publicly available, which currently incorporates only one-loop functionality, but an extension to two-loop cases is planned for future versions.
\end{abstract}




\section{Introduction}

For many production processes at colliders and other particle physics observables, the inclusion of higher-order radiative corrections is essential to match the experimental precision. Much progress towards efficient calculational techniques has been made over the last decades by many authors. However, the computation of loop diagrams with many different mass scales, many external legs, or more than one loop remains a difficult task. In particular, when going beyond the one-loop level, it is known that the loop integrals can in general not be solved analytically. Nevertheless, by using specialized semi-numerical techniques, several complete two-loop calculations have been carried out, for example for electroweak precision observables [1,2] and QCD corrections to the production of gauge bosons [3], top quarks [4] or Higgs bosons [5] at the Large Hadron Collider (LHC). However, the methods employed in these papers have been tailored to the problem at hand and cannot be applied easily to other situations.

Fully numerical techniques offer an alternative and potentially more flexible approach towards complex loop calculations. For a purely numerical method to become viable, two main obstacles need to be overcome: extracting the ultraviolet (UV) and infrared and collinear (IR) singularities from the integral; and ensuring robust and efficient convergence of the numerical integration. Several powerful methods have been proposed:

- Sector decomposition starts from the Feynman parametrization of a loop diagram. It isolates the physical singularities through appropriate mappings of the integration region [6]. There are additional integrable singularities inside the integration region for a diagram with physical cuts. While being formally integrable, such singularities cannot be handled by standard numerical integration algorithms. To avoid the problem and improve numerical stability one can deform the integration contours in the complex plane [7].

- The Feynman integrals can be transformed into Mellin-Barnes representations. An algorithm for the extraction of UV and IR poles has been developed based the residue theorem [8,9]. Again, the presence of physical cuts leads to bad convergence behavior of the numerical integrals, which can be improved by variable mapping and contour deformations [10].

- In a third approach the UV and IR singularities are removed from the integrand by suitable subtraction terms [11,12]. These subtraction terms are designed such that they can be easily integrated analytically and added back to the final result. As for the previous two methods, integrable singularities due to physical thresholds may be treated with the help of contour deformations [12].

The method based on subtraction terms has so far been applied only to one-loop calculations. This article presents a procedure for extending this idea to two-loop integrals. For this purpose the IR subtraction terms are constructed in analogy to Ref. [12,13] and combined with the contour deformation prescription of Ref. [7]. In addition, a new treatment for the UV divergences is introduced. As a first step, it is shown that this approach is already 


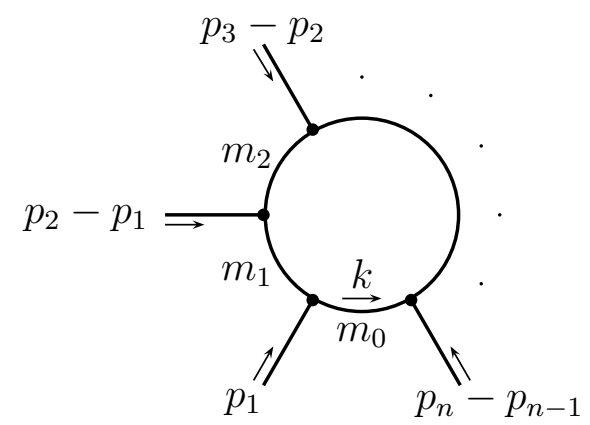

Figure 1: Topology of a general one-loop integral.

useful at the one-loop level, leading to numerical integrals with good convergence behavior. Secondly, it is demonstrated how it can be applied to two-loop diagrams. In the present paper, only two-loop integrals with IR singularities in one of the subloops are considered. The extension to overlapping IR singularities in both subloops will be delegated to a forthcoming publication [14, but it is relatively straightforward since most of the required subtraction terms can be constructed from the results of Ref. [15].

A public computer code for the evaluation of arbitrary one-loop integrals is provided, based on the method presented here. It is planned to incorporate two-loop cases into future versions of this code.

In the next section, the method for the numerical evaluation of one-loop integrals, using subtraction terms and contour deformation, is explained. It is demonstrated how it can be cast into an algorithmic form and its application in several examples is demonstrated. In section 3 the extension to the two-loop level is discussed and illustrated through a few examples. Finally, the main results are summarized in section 4 .

\section{One-loop integrals}

A general one-loop integral, see Fig. 1, may be written as

$$
\begin{aligned}
& I^{(1)}=\int \widetilde{d k} \frac{N(k)}{D^{(1)}(k)}, \quad \widetilde{d k}=e^{\gamma_{\mathrm{E}}(4-d) / 2} \frac{d^{d} k}{i \pi^{d / 2}}, \\
& D^{(1)}(k)=\left[k^{2}-m_{0}^{2}\right]\left[\left(k-p_{1}\right)^{2}-m_{1}^{2}\right] \cdots\left[\left(k-p_{n}\right)^{2}-m_{n}^{2}\right],
\end{aligned}
$$

where $N(k)$ is a polynomial in the loop momentum $k$. This integral may have soft, collinear and ultraviolet divergences. The first two are canceled in the integrand by using suitable subtraction terms, similar to Ref. [12] (see also Ref. [13]). However, no UV subtraction terms

are used for the one case here; instead the UV poles are evaluated explicitly after introducing Feynman parameters. 


\subsection{Infrared subtraction terms}

The $i$ th propagator of integral (2) produces a soft singularity if $m_{i}=0,\left(p_{i+1}-p_{i}\right)^{2}=m_{i+1}^{2}$, $\left(p_{i-1}-p_{i}\right)^{2}=m_{i-1}^{2}$, and $N(k) \neq 0$ for $k \rightarrow p_{i}$. The soft subtraction term is given by [12,13]

$$
\begin{aligned}
G_{\mathrm{soft}}^{(1)} & =\frac{1}{\left[\left(k-p_{i-1}\right)^{2}-m_{i-1}^{2}\right]\left[\left(k-p_{i}\right)^{2}\right]\left[\left(k-p_{i+1}\right)^{2}-m_{i+1}^{2}\right]} N\left(k=p_{i}\right) \prod_{\substack{j \neq \\
i-1, i, i+1}} \frac{1}{\left(p_{i}-p_{j}\right)^{2}-m_{j}^{2}} \\
& =\frac{1}{D^{(1)}(k)} N\left(k=p_{i}\right) \prod_{\substack{j \neq \\
i-1, i, i+1}} \frac{\left(k-p_{j}\right)^{2}-m_{j}^{2}}{\left(p_{i}-p_{j}\right)^{2}-m_{j}^{2}}
\end{aligned}
$$

where, for later convenience, the last line has been written in terms of the common denominator of eq. (2). The integrated soft subtraction term reads

$$
\begin{aligned}
& m_{i-1}=m_{i+1}=0: \quad \int \widetilde{d k} G_{\mathrm{soft}}^{(1)}=F_{i, \mathrm{rem}} \frac{1}{s}\left[\frac{1}{\varepsilon^{2}}-\frac{1}{\varepsilon} \log (-s)+\frac{\log ^{2}(-s)}{2}-\frac{\pi^{2}}{12}\right], \\
& m_{i-1}>0, m_{i+1}=0: \int \widetilde{d k} G_{\text {soft }}^{(1)}=F_{i, \text { rem }} \frac{1}{s-m_{i-1}^{2}}\left[\frac{1}{2 \varepsilon^{2}}-\frac{1}{\varepsilon} \log \left(\frac{m_{i-1}^{2}-s}{m_{i-1}}\right)+\frac{\pi^{2}}{24}\right. \\
& \left.+\frac{\log ^{2}\left(m_{i-1}^{2}-s\right)}{2}-\log ^{2}\left(m_{i-1}\right)-\operatorname{Li}_{2}\left(\frac{-s}{m_{i-1}^{2}-s}\right)\right] \\
& m_{i-1}, m_{i+1}>0 \text { : } \\
& \int \widetilde{d k} G_{\mathrm{soft}}^{(1)}=F_{i, \mathrm{rem}} \frac{-x}{m_{i-1} m_{i+1}\left(1-x^{2}\right)} \\
& \times\left[\log (x)\left(-\frac{1}{\varepsilon}-\frac{\log (x)}{2}+2 \log \left(1-x^{2}\right)+\log \left(m_{i-1} m_{i+1}\right)\right)\right. \\
& \left.-\frac{\pi^{2}}{6}+\mathrm{Li}_{2}\left(x^{2}\right)+\frac{1}{2} \log \left(\frac{m_{i-1}}{m_{i+1}}\right)+\mathrm{Li}_{2}\left(1+x \frac{m_{i-1}}{m_{i+1}}\right)+\mathrm{Li}_{2}\left(1+x \frac{m_{i+1}}{m_{i-1}}\right)\right], \\
& x=-\frac{1-\xi}{1+\xi}+i \epsilon, \quad \xi=\sqrt{1-\frac{4 m_{i-1} m_{i+1}}{s-\left(m_{i-1}-m_{i+1}\right)^{2}}},
\end{aligned}
$$

where $\varepsilon=(4-d) / 2, s=\left(p_{i+1}-p_{i-1}\right)^{2}+i \epsilon$, and

$$
F_{i, \mathrm{rem}}=N\left(k=p_{i}\right) \prod_{\substack{j \neq \\ i-1, i, i+1}} \frac{1}{\left(p_{i}-p_{j}\right)^{2}-m_{j}^{2}} .
$$

A collinear singularity singularity is encountered if $m_{i}=m_{i-1}=0,\left(p_{i}-p_{i-1}\right)^{2}=0$, and $N(k) \neq 0$ for $k \rightarrow p_{i}$. The simplest subtraction term for this singularity is given by

$$
\begin{aligned}
G_{\mathrm{coll}}^{(1)} & =\frac{1}{\left(k-p_{i-1}\right)^{2}\left(k-p_{i}\right)^{2}} N\left(k=p_{i}\right) \prod_{j \neq i-1, i} \frac{1}{\left(p_{i}-p_{j}\right)^{2}-m_{j}^{2}} \\
& =\frac{1}{D^{(1)}(k)} N\left(k=p_{i}\right) \prod_{j \neq i-1, i} \frac{\left(k-p_{j}\right)^{2}-m_{j}^{2}}{\left(p_{i}-p_{j}\right)^{2}-m_{j}^{2}}
\end{aligned}
$$


In dimensional regularization, the integrated collinear subtraction term is simply zero,

$$
\int \widetilde{d k} G_{\text {coll }}^{(1)}=0
$$

In other words, by subtracting $G_{\text {coll }}^{(1)}$ from the integrand the collinear singularity is transformed into a UV singularity, which will be handled as explained below. Note that this collinear subtraction term only works when applied to physical amplitude, i. e. to a gaugeinvariant set of diagrams [12].

\subsection{Variable mapping and contour deformation}

After subtracting the IR singularities as described above, one arrives at a loop integral that only contains UV divergences,

$$
I_{\text {reg }}^{(1)} \equiv I^{(1)}-\sum \int \widetilde{d k} G_{\text {soft }}^{(1)}-\sum \int \widetilde{d k} G_{\text {coll }}^{(1)}
$$

where the sums are over all soft and collinear singularities in $I^{(1)}$. It has a structure similar to eq. (2):

$$
I_{\mathrm{reg}}^{(1)}=\int \widetilde{d k} \frac{N_{\mathrm{reg}}(k)}{D^{(1)}(k)} .
$$

By introducing Feynman parameters and shifting the loop momentum, this expression can be cast into the form

$$
I_{\text {reg }}^{(1)}=\int d x_{1} \ldots d x_{n} \delta\left(1-\sum x_{i}\right) \int \widetilde{d k} \frac{\tilde{N}(k)}{\left[k^{2}-A\right]^{n}},
$$

where $\tilde{N}(k)$ is a polynomial in $k$ and in the Feynman parameters $x_{1}, \ldots, x_{n}$, while $A$ is a polynomial in $x_{1}, \ldots, x_{n}$. It is convenient to map the Feynman parameters onto a hypercube:

$$
\begin{aligned}
& x_{1}=1-y_{1}, \\
& x_{2}=y_{1}\left(1-y_{2}\right) \text {, } \\
& \int d x_{1} \ldots d x_{n} \delta\left(1-\sum x_{i}\right) \\
& x_{n-1}=y_{1} \cdots y_{n-2}\left(1-y_{n-1}\right), \quad=\int_{0}^{1} d y_{1} \ldots d y_{n} y_{1}^{n-2} y_{2}^{n-3} \cdots y_{n-2} \text {. } \\
& x_{n}=y_{1} \cdots y_{n-2} y_{n-1} \text {, }
\end{aligned}
$$

The $k$-dependence in the numerator of (13) is eliminated by using the tensor reduction formula

$$
\begin{aligned}
\int \widetilde{d k} & \frac{k^{\mu_{1}} k^{\mu_{2}} \cdots k^{\mu_{r}}}{\left[k^{2}-A\right]^{n}} \\
& =\frac{1}{r ! ! d(d+2) \cdots(d+r-2)} \sum_{\text {permut. }}\left(g^{\mu_{1} \mu_{2}} \cdots g^{\mu_{r-1} \mu_{r}}\right) \int \widetilde{d k} \frac{k^{r}}{\left[k^{2}-A\right]^{n}},
\end{aligned}
$$


where the sum is over all permutations of $\mu_{1}, \ldots, \mu_{r}$. Thus one arrives at

$$
I_{\text {reg }}^{(1)}=\int_{0}^{1} d y_{1} \ldots d y_{n-1} \int \widetilde{d k}\left[\frac{C_{1}}{\left[k^{2}-A\right]^{n}}+\frac{C_{2}}{\left[k^{2}-A\right]^{n-1}}+\ldots\right],
$$

where the $C_{i}$ are polynomials in $y_{1}, \ldots, y_{n}$ but independent of $k$. Integrating over $k$, one obtains

$$
\begin{aligned}
I_{\text {reg }}^{(1)}=\int_{0}^{1} d y_{1} \ldots d y_{n-1} & {\left[C_{1}(-1)^{n} \frac{\Gamma(n+\varepsilon-2)}{\Gamma(n)} A^{-n-\varepsilon+2}\right.} \\
& \left.+C_{2}(-1)^{n-1} \frac{\Gamma(n+\varepsilon-3)}{\Gamma(n-1)} A^{-n-\varepsilon+3}+\ldots\right],
\end{aligned}
$$

which can be expanded in $\varepsilon$, so that the UV singularities appear as $1 / \varepsilon$ poles. Thus the integral takes the form

$$
I_{\text {reg }}^{(1)}=\int_{0}^{1} d y_{1} \ldots d y_{n-1}\left[D_{0}\left(\frac{1}{\varepsilon}+\log (A-i \epsilon)\right)+D_{1}(A-i \epsilon)^{-1}+D_{2}(A-i \epsilon)^{-2}+\ldots\right],
$$

where the $i \epsilon$ from the propagators have been made explicit again. $A$ and the $D_{i}$ are polynomials in the variables $y_{1}, \ldots, y_{n-1}$.

The coefficients in the $\varepsilon$-expansion in eq. (18) are finite, so that the integration over the parameters $y_{1}, \ldots, y_{n-1}$ can be performed numerically. However, the polynomial $A(\vec{y})$ can have zeros inside the integration region, which happens if the loop integral has physical thresholds. While these singularities are formally integrable, they lead to problems for the numerical integration. A solution is the deformation of the integration contours into the complex plane, by using the variable transformation [7]

$$
y_{i}=z_{i}-i \lambda z_{i}\left(1-z_{i}\right) \frac{\partial A}{\partial z_{i}}, \quad 0 \leq z_{i} \leq 1
$$

To leading order in $\lambda$ this produces a negative imaginary part in $A$ :

$$
A(\vec{y})=A(\vec{z})-i \lambda \sum_{i} z_{i}\left(1-z_{i}\right)\left(\frac{\partial A}{\partial z_{i}}\right)^{2}+\mathcal{O}\left(\lambda^{2}\right) .
$$

So if $\lambda$ is chosen small enough, the integral

$$
I_{\text {reg }}^{(1)}=\int_{0}^{1} d z_{1} \ldots d z_{n-1}\left|\frac{\partial\left(y_{1}, \ldots, y_{n-1}\right)}{\partial\left(z_{1}, \ldots, z_{n-1}\right)}\right|\left[D_{0}\left(\frac{1}{\varepsilon}+\log (A-i \epsilon)\right)+D_{1} A^{-1}+D_{2} A^{-2}+\ldots\right]
$$

is well-behaved for numerical integration. In most cases, a good choice for $\lambda$ is roughly 0.5.

\subsection{Numerical examples}

To demonstrate the flexibility and efficiency of the method described in the previous section, several example calculations have been carried out and, where applicable, compared to existing analytical results.

The numerical integration has been performed with the VEGAS and CUHRE algorithms of the CuBA 1.4 library [18]. Timing information is given for running on a single core of a INTEL $^{\circledR} \mathrm{XEON}^{\circledR}$ X5570 processor with $2.93 \mathrm{GHz}$. 


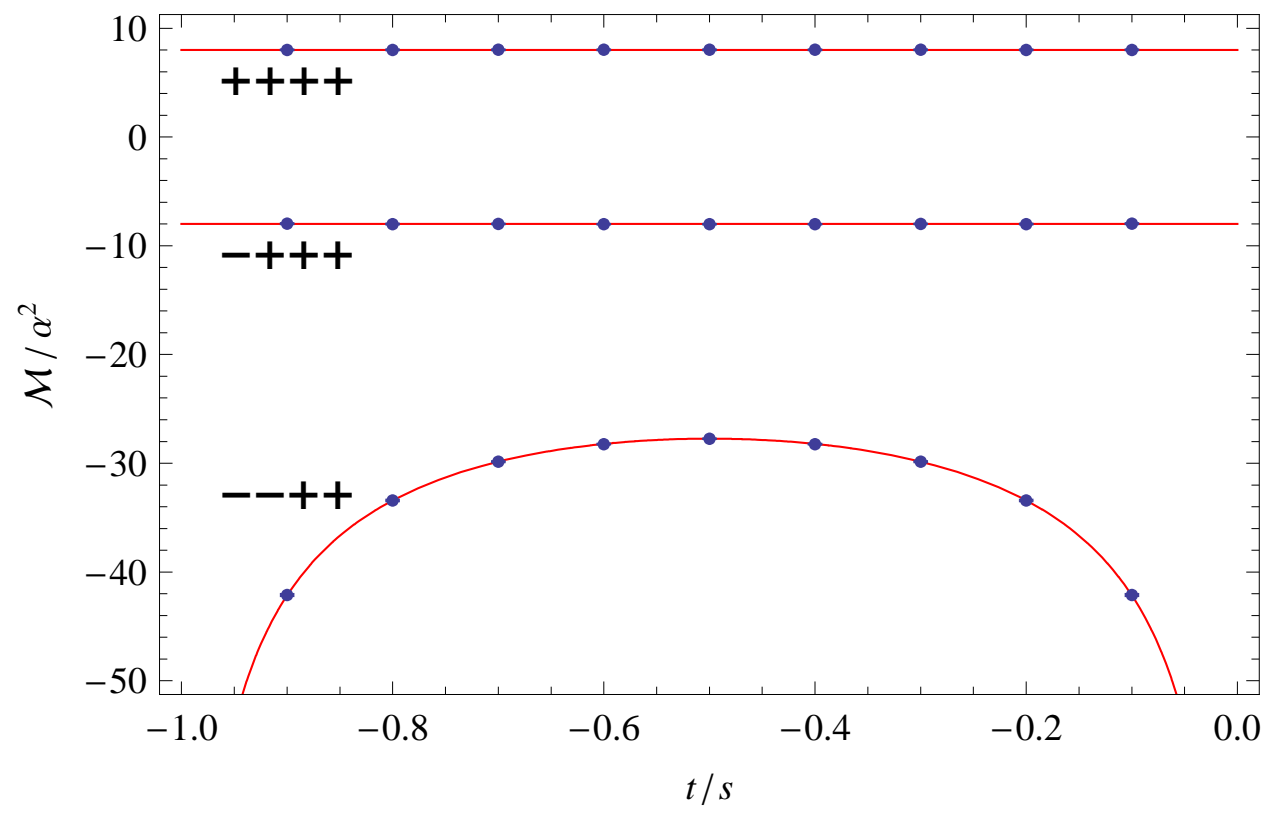

Figure 2: Results for the four-photon amplitude $\mathcal{M}(\gamma \gamma \rightarrow \gamma \gamma$ ) (normalized relative to $\left.\alpha^{2}\right)$ for different photon helicity combinations. The dots correspond to the results obtained with the numerical method presented here, while the lines depict the analytical result from Ref. [21]. The contour deformation parameter has been chosen $\lambda=1$.
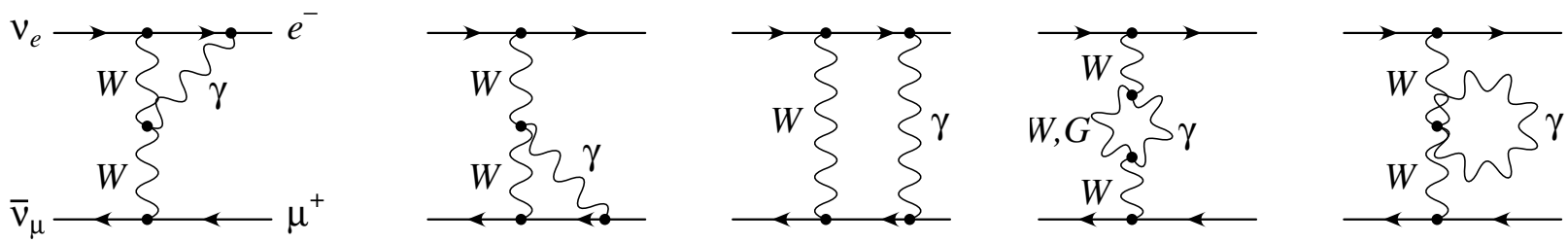

Figure 3: Feynman diagrams for the one-loop QED corrections to $\nu_{e} \bar{\nu}_{\mu} \rightarrow e^{-} \mu^{+}$.

1. Let us start with the amplitude for two-photon scattering $\gamma \gamma \rightarrow \gamma \gamma$ at the one-loop level, with electrons running in the loop. This process does not involve any physical singularities and thus is a simple test of the contour deformation and numerical integration. The six contributing diagrams are grouped into three groups, according to the structure of the loop denominators. Each group is evaluated separately, using the VEGAS algorithm with $10^{6}$ integration points each, which in total takes $4.8 \mathrm{~s}$ evaluation time. Results for different helicity combinations of the photons are depicted in Fig. 2, and compared to analytical results from Ref. [21]. The numerical integration error ranges between $0.2 \%$ and $0.5 \%$; error bars are included but not visible in the plot.

2. The one-loop QED corrections to $\nu_{e} \bar{\nu}_{\mu} \rightarrow e^{-} \mu^{+}$is an example with UV, soft and collinear singularities. The six diagrams in Fig. 3 are combined into a single expression with one common denominator, from which the soft and collinear divergences are subtracted as explained in section 2.1. 


\begin{tabular}{lcc}
\hline & \multicolumn{1}{c}{ this work } & \multicolumn{1}{c}{ analytical } \\
\hline $\mathcal{O}\left(\varepsilon^{-2}\right)$ & -0.625 & -0.625 \\
$\mathcal{O}\left(\varepsilon^{-1}\right)$ & $1.13113365-1.96349541 i$ & $1.13113365-1.96349541 i$ \\
$\mathcal{O}\left(\varepsilon^{0}\right)$ & $3.27791(4)+1.83014(4) i$ & $3.27792343+1.83014477 i$ \\
\hline
\end{tabular}

Table 1: Numerical results for the next-to-leading order QED corrections to the squared matrix element of $\nu_{e} \bar{\nu}_{\mu} \rightarrow e^{-} \mu^{+}$. Specifically, the table shows numbers for $2 \operatorname{Re}\left\{\mathcal{M}^{(0)} \mathcal{M}^{(1)}\right\}$, for the input parameters $M_{\mathrm{W}}=1, s=2, t=-1$, and $\lambda=0.5$. The results from the numerical method described in this paper are given in the second column, together with the numerical error from $5 \times 10^{5}$ integration points of the CuHRE integration routine. For comparison the third column lists the results obtained with the traditional analytical approach using Passarino-Veltman reduction.

The results presented in Tab 1 correspond to the next-to-leading order correction to the squared matrix element, given by $2 \operatorname{Re}\left\{\mathcal{M}^{(0)} \mathcal{M}^{(1)}\right\}$, where $\mathcal{M}^{(0)}$ and $\mathcal{M}^{(1)}$ refer to the treelevel and one-loop QED corrected matrix elements, respectively. The numerical integration has been performed with the CUHRE algorithm, using $5 \times 10^{5}$ integration points (resulting in $2.4 \mathrm{~s}$ running time). Note that the $\varepsilon^{-2}$ pole stems solely from the soft subtraction term and thus is known analytically. For comparison, the table also shows the result from a traditional analytical calculation using Passarino-Veltman reduction [19] and explicit expression for the well-known basic integrals, see e.g. Ref. [20].

3. A third example, with a more difficult denominator structure, is the scalar hexagon integral depicted in Fig. 4, It contains several mass scales, has three soft singularities, and requires contour deformation. Numerical results are shown for the input values

$$
\begin{array}{lll}
m_{\mathrm{a}}^{2}=1.0, & \vec{p}_{1}=(0,0,3), & \vec{p}_{2}=(0,0,-3), \\
m_{\mathrm{b}}^{2}=0.25, & \vec{p}_{3}=(0.5,0,0.5), & \vec{p}_{4}=(-0.2,0,0.1), \\
m_{\mathrm{c}}^{2}=4.0, & \vec{p}_{5}=(0,1.37626,-0.3), & \overrightarrow{p_{6}}=(-0.3,-1.37626,-0.3),
\end{array}
$$

and $\lambda=0.5$. Using $10^{7}$ integration points with the CUHRE algorithm one obtains

$$
\begin{array}{ll}
\mathcal{O}\left(\varepsilon^{-1}\right): & -0.0044718804+0.0120697975 i, \\
\mathcal{O}\left(\varepsilon^{0}\right): & -0.04383(14)+0.00790(14) i,
\end{array}
$$

which takes 45 min to evalute on the test computer used here.

\subsection{The computer package NICODEMOS}

The procedure outlined in sections 2.1 and 2.2 has been implemented into the public computer code NICODEmos (Numerical Integration with COntour DEformation and MOdular Subtractions). The package contains a Mathematica module, which performs the application of subtraction terms and Feynman parametrization (with or without deformation). 


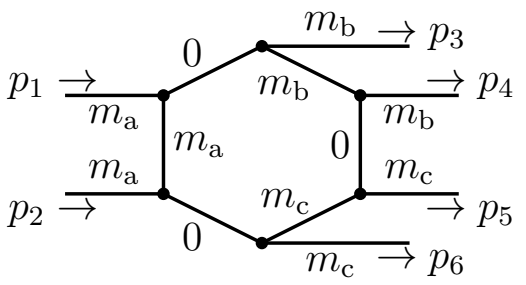

Figure 4: Scalar hexagon diagram with soft singularities, labeling the masses of all internal and external lines and the external momenta.

The user needs to supply the input expression and the location of IR singularities. The MATHEMATICA code then produces a FORTRAN executable, which performs the numerical integration for a given set of numerical input parameters.

NICODEMOS is available for download at http://www.pitt.edu/ ªfreitas/ and can be used freely, provided the source is acknowledged and properly cited. Version 1.0 can only handle one-loop integrals, but it is planned to expand the functionality to include two-loop integrals in future versions.

\section{Two-loop integrals}

In this section the extension to two-loop diagrams is discussed. The two-loop integrals may contain UV singularities as well as IR singularities in one subloop, while the case with IR singularities in both subloops will be addressed in a future publication [14].

Of particular interest are relatively complex two-loop diagrams, i.e. diagrams with a relatively large number of external legs. Therefore the following discussion will not consider some special cases that occur only for two-loop tadpole and selfenergy diagrams, since these can be evaluated with existing methods, see e.g. Ref. [22].

Following the notation of the previous section, a two-loop integral is given by

$$
\begin{aligned}
I^{(2)}=\int \widetilde{d k_{1}} \widetilde{d k}_{2} & \frac{N\left(k_{1}, k_{2}\right)}{D^{(2)}\left(k_{1}, k_{2}\right)}, \\
D^{(2)}\left(k_{1}, k_{2}\right)= & {\left[k_{1}^{2}-m_{0}^{2}\right]\left[\left(k_{1}-p_{1}\right)^{2}-m_{1}^{2}\right] \cdots\left[\left(k_{1}-p_{r}\right)^{2}-m_{r}^{2}\right] } \\
& \times\left[\left(k_{2}-p_{r+1}\right)^{2}-m_{r+1}^{2}\right] \cdots\left[\left(k_{2}-p_{s}\right)^{2}-m_{s}^{2}\right] \\
& \times\left[\left(k_{1}-k_{2}-p_{s+1}\right)^{2}-m_{s+1}^{2}\right] \cdots\left[\left(k_{1}-k_{2}-p_{n}\right)^{2}-m_{n}^{2}\right],
\end{aligned}
$$

where $N\left(k_{1}, k_{2}\right)$ is polynomial in $k_{1}$ and $k_{2}$ and in the external momenta.

If one of the two subloops has an IR singularity it can be subtracted analogously to the one-loop case. For example, the scalar diagram in Fig. 5

$$
\begin{aligned}
I_{\mathrm{fig}[5]} & =\int \widetilde{d k}_{1} \widetilde{d k}_{2} \\
& \times \frac{1}{\left[k_{1}-m^{2}\right]\left[\left(k_{1}-p_{1}\right)^{2}\right]\left[\left(k_{1}-p_{1}-p_{2}\right)^{2}-m^{2}\right]\left[\left(k_{2}-p_{1}\right)^{2}-m^{2}\right]\left[\left(k_{1}-k_{2}\right)^{2}-m^{2}\right]}
\end{aligned}
$$

has a soft singularity for $k_{1} \rightarrow p_{1}$. The subtraction term is constructed as in eq. (3), viz. 


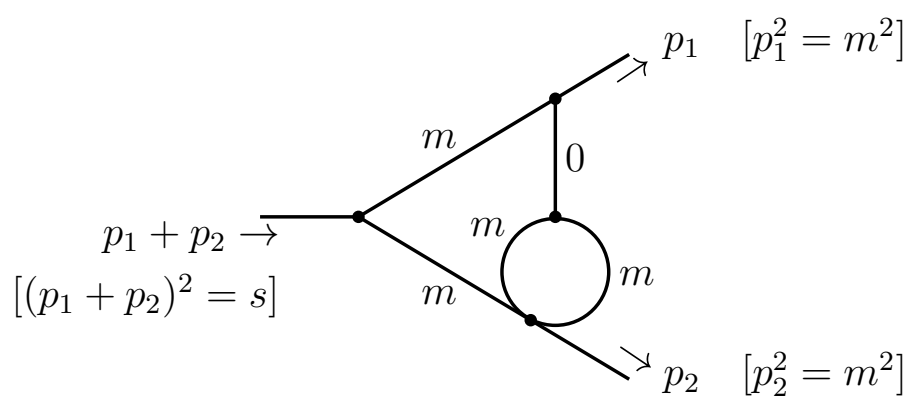

Figure 5: Scalar two-loop diagram with soft singularity. The figures also labels the masses of all internal and external lines and the external momenta.

$$
G_{\mathrm{soft}}^{\mathrm{fid} 5]}=\frac{1}{\left[k_{1}-m^{2}\right]\left[\left(k_{1}-p_{1}\right)^{2}\right]\left[\left(k_{1}-p_{1}-p_{2}\right)^{2}-m^{2}\right]\left[\left(k_{2}-p_{1}\right)^{2}-m^{2}\right]\left[\left(p_{1}-k_{2}\right)^{2}-m^{2}\right]} .
$$

This expression factorizes into two one-loop integrals, which can be integrated analytically.

In the same way, one can subtract collinear divergences in one subloop with the subtraction terms introduced in section 2.1 .

\subsection{Ultraviolet divergences}

Since two-loop integrals may have overlapping UV singularities from both subloops, the corresponding $1 / \varepsilon$ poles cannot be computed directly as in eq. (18). Instead one has to introduce subtraction terms also for the UV divergences. The UV subtraction is performed in two steps:

1. The global UV singularities of both subloops can be obtained by performing a Taylor expansion of the two-loop amplitude in terms of the external momenta. For all physical amplitudes with three or more external legs only the leading term in this expansion contributes to the global UV divergence. Two-loop tadpoles and selfenergies are not considered here, as mentioned above. Thus the global UV subtraction term is defined as

$$
G_{\text {glob }}^{(2)}=\left.\frac{N\left(k_{1}, k_{2}\right)}{D^{(2)}\left(k_{1}, k_{2}\right)}\right|_{p_{i}=0}
$$

The integrated subtraction term $\int \widetilde{d k_{1}} \widetilde{d k}_{2} G_{\text {glob }}^{(2)}$ consists of two-loop vacuum integrals, which can be evaluated analytically with the methods of Ref. [23].

2. The remainder $I_{\mathrm{gs}}^{(2)} \equiv I_{\mathrm{reg}}^{(2)}-\int \widetilde{d k}_{1} \widetilde{d k}_{2} G_{\mathrm{glob}}^{(2)}$ can still contain a UV singularity in one of the subloops, or in both. The latter case, however, only occurs for tadpole and selfenergy diagrams and thus will not be considered here, since it can be handled more efficiently with other methods [22].

Let us then assume that only the subloop with loop momentum $k_{1}$ has a UV divergence. The $k_{1}$ integral is now turned into a Feynman-parameter integral by following the steps in 


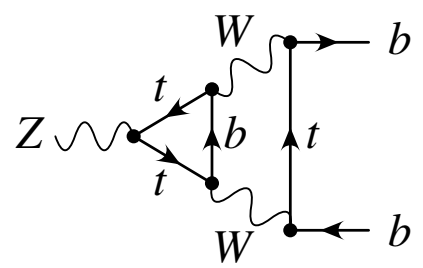

Figure 6: Typical diagram for the two-loop correction to the $Z b \bar{b}$ vertex.

eqs. (13) $-(16)$, leading to

$$
I_{\mathrm{gs}}^{(2)}=\int_{0}^{1} d y_{1} \ldots d y_{m-1} \int \widetilde{d k_{1}} \widetilde{d k_{2}}\left[\frac{C_{1}}{\left[k_{1}^{2}-A\right]^{m}}+\frac{C_{2}}{\left[k_{1}^{2}-A\right]^{m-1}}+\ldots\right],
$$

where the $C_{i}$ and $A$ depend on $k_{2}$ and the Feynman parameters $y_{1}, \ldots, y_{m-1}$. Here $m=n+r-s$ is the number of propagators with $k_{1}$. Note that the $C_{i}$ are rational functions containing the propagators that depend on $k_{2}$ only. At this point, a subloop UV singularity is indicated by a term of the form $C_{j} /\left[k_{1}^{2}-A\right]^{2}$ in eq. (29). All higher powers of $\left[k_{1}^{2}-A\right]$ in the denominator are UV-finite. The subloop UV divergence can be subtracted by

$$
G_{\mathrm{sub}}^{(2)}=\int_{0}^{1} d y_{1} \ldots d y_{m-1} \frac{C_{j}}{\left[k_{1}^{2}-\mu^{2}\right]^{2}}
$$

where $\mu$ is a suitably chosen mass parameter. The integrated subtraction term factorizes into two one-loop integrals:

$$
\int \widetilde{d k_{1}} \widetilde{d k}_{2} G_{\mathrm{sub}}^{(2)}=-\Gamma(\varepsilon-2) \mu^{2-\varepsilon} \int_{0}^{1} d y_{1} \ldots d y_{m-1} \int \widetilde{d k}_{2} C_{j}
$$

The one-loop integral over $k_{2}$ can now be carried out with the procedure of section 2.2 .

The remaining two-loop integral $I_{\text {rem }}^{(2)} \equiv I_{\text {reg }}^{(2)}-\int \widetilde{d k_{1}} \widetilde{d k}_{2} G_{\text {glob }}^{(2)}-\int \widetilde{d k_{1}} \widetilde{d k}_{2} G_{\text {sub }}^{(2)}$ is finite. After introducing Feynman parameters for the $k_{2}$ integral, which are mapped onto a hypercube, and shifting the $k_{2}$ loop momentum one arrives at an expression of the form

$$
I_{\text {rem }}^{(2)}=\int_{0}^{1} d y_{1} \ldots d y_{n-1} \int \widetilde{d k_{1}} \widetilde{d k}_{2}\left[\frac{\tilde{C}_{1}\left(k_{2}\right)}{\left[\alpha k_{1}^{2}+\beta k_{2}^{2}-\tilde{A}\right]^{n}}+\frac{\tilde{C}_{2}\left(k_{2}\right)}{\left[\alpha k_{1}^{2}+\beta k_{2}^{2}-\tilde{A}\right]^{n-1}}+\cdots\right]
$$

where $\alpha, \beta$ and $\tilde{A}$ are polynomials in the Feynman parameters $y_{1}, \ldots, y_{n-1}$, and $\tilde{C}_{i}\left(k_{2}\right)$ are polynomials in $k_{2}$ and the Feynman parameters. Now one can perform the tensor reduction for $k_{2}$, the $k_{2}$ loop integration, and-if necessary - the contour deformation in analogy to eqs. (15), (17), and (19).

\subsection{Numerical examples}

1. A typical example with global and subloop UV singularities is given by the diagram in Fig. 6, which contributes to the two-loop corrections to the effective weak mixing angle of 


\begin{tabular}{lcc}
\hline & this work & \multicolumn{1}{c}{ Ref. [2] } \\
\hline $\mathcal{O}\left(\varepsilon^{-2}\right)$ & -2.30183413 & -2.30183413 \\
$\mathcal{O}\left(\varepsilon^{-1}\right)$ & 5.07108758 & 5.07108758 \\
$\mathcal{O}\left(\varepsilon^{0}\right)$ & $8.326(1)$ & 8.3259 \\
\hline
\end{tabular}

Table 2: Numerical results for the contribution of the diagram in Fig. 6 to the effective weak mixing angle $\sin ^{2} \theta_{\mathrm{eff}}^{b \bar{b}}$, in units of $(\alpha / 4 \pi)^{2}$. The input parameters are chosen as in Ref. [2]: $M_{\mathrm{Z}}=1, M_{\mathrm{W}}=80 / 91, m_{\mathrm{t}}=180 / 91$. The deformation parameter is set to $\lambda=0$. The numbers in the second column have been obtained using $10^{6}$ integration points of the CuHRe integration routine, with the integration error given in brackets. The last column shows the results from to Tab. 1 in Ref. [2] for comparison.

\begin{tabular}{lll}
\hline & \multicolumn{1}{c}{ this work } & \multicolumn{1}{c}{ Ref. [15] } \\
\hline $\mathcal{O}\left(\varepsilon^{-2}\right)$ & $-0.43040894+1.40496295 i$ & $-0.43040894+1.40496295 i$ \\
$\mathcal{O}\left(\varepsilon^{-1}\right)$ & -3.53105702 & -3.53105702 \\
$\mathcal{O}\left(\varepsilon^{0}\right)$ & $-1.93471(1)-2.08763(1) i$ & $-1.93471213-2.08762578 i$ \\
\hline
\end{tabular}

Table 3: Results for the numerical evaluation of the scalar diagram in Fig. 15. The second column shows the values obtained with the method of this paper, using $m=1, s=5, \lambda=1$ and $10^{6}$ integration points of the CuHRE algorithm. For comparison, the last column gives the analytical result from eq. (343) in Ref. [15].

bottom quarks, $\sin ^{2} \theta_{\text {eff }}^{b \bar{b}}$. Since this diagram does not have any physical cuts, no deformation of the integration contour is needed and one can choose $\lambda=0$. Tab. 2 shows the numerical results obtained with the algorithm of the paper, using $10^{6}$ integration points of the CuHRE routine. The final answer is the sum of the global UV subtraction term, the subloop UV subtraction term and the remaining finite two-loop integral. The integration error is negligible except for the finite $\mathcal{O}\left(\varepsilon^{0}\right)$ term. The numerical integration takes about 13 min to evaluate on one core of a INTEL ${ }^{\circledR}$ XEON ${ }^{\circledR}$ X5570 processor with $2.93 \mathrm{GHz}$. For comparison, the table also shows the result of Ref. [2], which has been obtained with the Bernstein-Tkachov (BT) method. The two results agree very well within numerical integration errors.

2. Fig. [5] shows an example of a scalar diagram with both UV and IR divergences. The soft subtraction in eq. (27) is used, but its integrated form needs to be evaluated to order $\mathcal{O}(\varepsilon)$, due to the presence of the UV singularity. Numerical results obtained with this method are shown in the second column of Tab. 3, using $10^{6}$ integration points of the CuHRE routine, corresponding to a running time of $2.6 \mathrm{~s}$. For this particular diagram an analytical result has been obtained previously in terms of harmonic polylogarithms [15], which is also shown in the table for comparison. 


\section{Summary}

This paper presents a procedure for numerically computing one- and two-loop integrals using subtraction terms for the singular pieces of the integrand. A set of subtraction terms for the removal of ultraviolet, soft and collinear singularities has been described. The subtraction terms themselves can be easily integrated analytically in dimensional regularization, while the difference between the full loop amplitude and the subtracted contributions is finite and can be evaluated numerically after taking the limit to four dimensions. The approach is applied to general one-loop cases, as well as two-loop integrals with global and subloop ultraviolet divergences but infrared divergences in only one of the two subloops. The extension to overlapping infrared singularities in both subloops will be discussed in a subsequent publication.

The finite numerical integral (after application of the subtraction terms and expansion in the integration dimension) is evaluated in Feynman parameter space. The integrand can still have singular points inside the integration regions if the diagram has physical thresholds. These singularities are formally integrable but are problematic for numerical integration routines, so that they must be avoided by deforming the integration contour into the complex plane.

The usefulness of the proposed procedure has been demonstrated with several one- and two-loop examples. One obtains an overall good convergence behavior of the numerical integration, although problems can occur for integrals with pinch singularities, which typically correspond to threshold configurations. It may be possible to regularize the pinch singularities with additional finite subtraction terms, but a detailed investigation of this matter is left for future work.

The algorithm presented in this paper has been implemented in the public computer program NICODEMOS (available at http://www.pitt.edu/ afreitas/). It is based on MATHEMATICA for symbolic manipulations and produces a FORTRAN executable for the numerical evaluation. The current version 1.0 only contains one-loop functionality, but an extension to the two-loop level is planned for future releases.

\section{Acknowledgements}

This work has been supported in part by the National Science Foundation under grant no. PHY-0854782.

\section{References}

[1] A. Freitas, W. Hollik, W. Walter and G. Weiglein, Phys. Lett. B 495, 338 (2000) [Erratum-ibid. B 570, 260 (2003)];

M. Awramik, M. Czakon, A. Freitas and G. Weiglein, Phys. Rev. D 69 (2004) 053006;

M. Awramik, M. Czakon, A. Freitas, G. Weiglein, Phys. Rev. Lett. 93, 201805 (2004);

M. Awramik, M. Czakon and A. Freitas, Phys. Lett. B 642, 563 (2006); 
W. Hollik, U. Meier and S. Uccirati, Nucl. Phys. B 731, 213 (2005);

M. Awramik, M. Czakon and A. Freitas, JHEP 0611, 048 (2006);

W. Hollik, U. Meier and S. Uccirati, Nucl. Phys. B 765, 154 (2007);

A. Freitas and Y. C. Huang, arXiv:1205.0299.

[2] M. Awramik, M. Czakon, A. Freitas and B. A. Kniehl, Nucl. Phys. B 813, 174 (2009).

[3] R. Hamberg, W. L. van Neerven and T. Matsuura, Nucl. Phys. B 359, 343 (1991) [Erratum-ibid. B 644, 403 (2002)];

C. Anastasiou, L. Dixon, K. Melnikov and F. Petriello, Phys. Rev. D 69, 094008 (2004);

K. Melnikov and F. Petriello, Phys. Rev. D 74, 114017 (2006).

[4] M. Czakon, A. Mitov and S. Moch, Phys. Lett. B 651, 147 (2007);

M. Czakon, A. Mitov and S. Moch, Nucl. Phys. B 798, 210 (2008);

M. Czakon, Phys. Lett. B 664, 307 (2008);

? R. Bonciani, A. Ferroglia, T. Gehrmann, D. Maître and C. Studerus, ? JHEP 0807, 129 (2008);

? R. Bonciani, A. Ferroglia, T. Gehrmann and C. Studerus, JHEP 0908, 067 (2009);

P. Baernreuther, M. Czakon and A. Mitov, arXiv:1204.5201.

[5] R. V. Harlander and W. B. Kilgore, Phys. Rev. Lett. 88, 201801 (2002);

C. Anastasiou and K. Melnikov, Nucl. Phys. B 646, 220 (2002);

V. Ravindran, J. Smith and W. L. van Neerven, Nucl. Phys. B 665, 325 (2003);

C. Anastasiou, K. Melnikov and F. Petriello, Phys. Rev. Lett. 93, 262002 (2004);

G. Davatz et al., JHEP 0607, 037 (2006);

S. Catani and M. Grazzini, Phys. Rev. Lett. 98, 222002 (2007);

S. Actis, G. Passarino, C. Sturm and S. Uccirati, Phys. Lett. B 670, 12 (2008). ?

[6] T. Binoth and G. Heinrich, Nucl. Phys. B 585, 741 (2000);

T. Binoth and G. Heinrich, Nucl. Phys. B 680, 375 (2004);

J. Carter and G. Heinrich, Comput. Phys. Commun. 182, 1566 (2011).

[7] Z. Nagy and D. E. Soper, Phys. Rev. D 74, 093006 (2006);

C. Anastasiou, S. Beerli and A. Daleo, JHEP 0705, 071 (2007);

S. Borowka, J. Carter and G. Heinrich, arXiv:1204.4152.

[8] C. Anastasiou and A. Daleo, JHEP 0610, 031 (2006).

[9] M. Czakon, Comput. Phys. Commun. 175, 559 (2006).

[10] A. Freitas and Y. C. Huang, JHEP 1004, 074 (2010).

[11] Z. Nagy and D. E. Soper, JHEP 0309, 055 (2003).

[12] S. Becker, C. Reuschle and S. Weinzierl, JHEP 1012, 013 (2010).

[13] S. Dittmaier, Nucl. Phys. B 675, 447 (2003). 
[14] A. Freitas, in preparation.

[15] R. Bonciani, P. Mastrolia and E. Remiddi, Nucl. Phys. B 661, 289 (2003) [Erratum-ibid. B 702, 359 (2004)].

[16] G. P. Lepage, "Vegas: An Adaptive Multidimensional Integration Program," report CLNS-80/447 (1980).

[17] Wolfram Research Inc., "Mathematica Version 6.0" (2007) [http://www.wolfram.com/products/mathematica/newin6/].

[18] T. Hahn, Comput. Phys. Commun. 168, 78 (2005).

[19] G. Passarino and M. J. G. Veltman, Nucl. Phys. B 160, 151 (1979).

[20] W. Beenakker and A. Denner, Nucl. Phys. B 338, 349 (1990);

R. K. Ellis and G. Zanderighi, JHEP 0802, 002 (2008).

[21] T. Binoth, E. W. N. Glover, P. Marquard and J. J. van der Bij, JHEP 0205, 060 (2002).

[22] G. Weiglein, R. Scharf and M. Böhm, Nucl. Phys. B 416, 606 (1994);

S. Bauberger, F. A. Berends, M. Böhm and M. Buza, Nucl. Phys. B 434, 383 (1995).

[23] A. I. Davydychev and J. B. Tausk, Nucl. Phys. B 397, 123 (1993). 


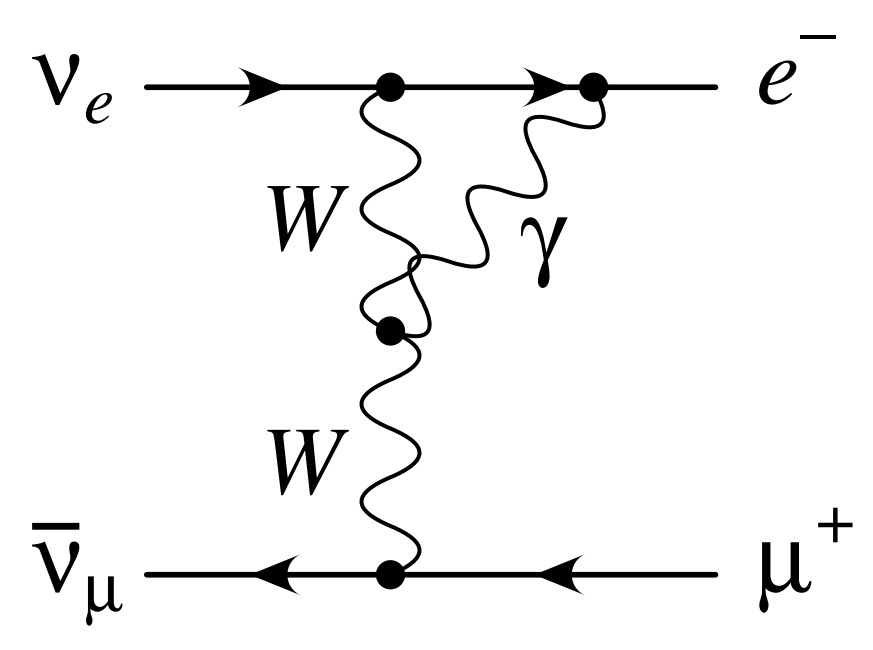

